\title{
Cationic Surface Modification of Cellulose Nanocrystals: Toward Tailoring Dispersion and Interface in Carboxymethyl Cellulose Films
}

Mei-Chun $\mathrm{Li}^{\mathrm{a}}$, Changtong Mei ${ }^{\mathrm{b}, *}$, Xinwu Xu ${ }^{\mathrm{b}}$, Sunyoung Lee ${ }^{\mathrm{c}}$, and Qinglin $\mathrm{Wu}^{\mathrm{a}}{ }^{*}$

${ }^{a}$ School of Renewable Natural Resources, Louisiana State University AgCenter, Baton Rouge, Louisiana 70803, USA

${ }^{\mathrm{b}}$ College of Materials Science and Engineering, Nanjing Forestry University, Nanjing, 210037, China

c Department of Forest Products, Korea National Institute of Forest Science, Seoul, 130712, Republic of Korea

* Corresponding Authors. E-mails: wuqing@1su.edu (Q.wu); mei@njfu.edu.cn (C. Mei) 


\begin{abstract}
The present work describes the development of high performance cellulose nanocrystals/carboxymethyl cellulose (CNC/CMC) films through a rational design of CNC surface chemistry. Considering the anionic surface nature of CMC, surface cationization of CNCs was performed in order to build strong interfacial bonding by electrostatic attraction and form uniform dispersion state by electrostatic repulsion. Nanocomposite films were fabricated by dispersing the CNCs and cationically modified CNCs (mCNCs) in CMC matrix through solution casting. The resultant $\mathrm{CNC} / \mathrm{CMC}$ and $\mathrm{mCNC} / \mathrm{CMC}$ films were then evaluated in terms of processability, mechanical properties and fracture surface morphology. Results showed that the reinforcing capacity of mCNCs was greatly dependent on its substitution degree. High substitution degree was favorable for obtaining $\mathrm{mCNC} / \mathrm{CMC}$ film with unifrom dispersion and superior mechanical properties. The observed distinctive reinforcing phenomena from CNCs and mCNCs in CMC matrix were interpreted in terms of dispersion state and interfacial bonding based on the fracture surface morphology.
\end{abstract}

Keywords: Carboxymethyl cellulose, Cellulose nanocrystals, Surface cationization, Interfacial interaction, Mechanical properties. 


\section{Introduction}

In the past decades, great efforts have been made in order to achieve the environmental protection and sustainable development goals [1]. One of the most economic and efficient approaches to realize these goals is the utilization of biopolymers as an alternative to petroleumbased polymers due to their low cost, abundant, renewable, environmentally friendly, biocompatible and biodegradable advantages [2,3]. Carboxymethyl cellulose (CMC) is an anionic cellulose derivative produced by the alkali-catalyzed reaction of cellulose with chloroacetic acid. The introduction of carboxymethyl and hydroxyl groups on the surface of CMC makes it highly soluble in water and chemically reactive. Because of its appealing intrinsic characteristics, i.e., odorless, ionic nature, thickening, stabilizing, thermal gelation and water retention, CMC has broad applications in the various fields, e.g., food, detergent, toothpaste, drilling fluids, adhesives, biomedicines and cosmetics [4].

Recently, the development of low cost, sustainable, and high performance biopolymer films using carboxymethyl cellulose (CMC) has attracted much attention from both academic and industrial researchers due to its transparency, nontoxicity, biocompatibility, biodegradability and good film forming processability [5-13]. Unfortunately, the poor physicochemical performance of CMC greatly limited its wide application under different environmental conditions. One of the most effective methods to overcome these bottlenecks is the incorporation of reinforcing fillers. It was reported that various nanoscale fillers, e.g., graphene oxide [7], nanoclay [8], layered double hydroxide [9], chitin nanofibers [10] and cellulose nanocrystals (CNCs) [11-13] exhibited effective reinforcement in CMC films owing to extremely large surface area and aspect ratio. Considering the environmental protection and sustainable development issues, the use of green, renewable and biodegradable CNCs is highly preferred. 
For example, Oun and Rihm [13] extracted three types of CNCs from rice straw, wheat straw and barley straw and used them as fillers in CMC films. It was found that the presence of CNCs effectively improved the mechanical and water vapor barrier performance of CMC films. Similar improvements were also achieved through the incorporation of $\mathrm{CNCs}$ isolated from sugarcane bagasse in starch/CMC composite films [12].

It is usually accepted that the dispersion state of $\mathrm{CNCs}$ in polymer matrix, the interface interaction between $\mathrm{CNCs}$ and polymer matrix as well as the formation of $\mathrm{CNC}$ percolating network within the polymer matrix are the three most critical factors determining the reinforcement of CNCs in polymer matrix [14,15]. Only when the CNCs are homogeneously dispersed in polymer matrix, their advantages, i.e., large aspect ratio, high modulus and large surface area to interact with polymer matrix, can be fully realized. In addition, the strong adhesion occurring at the CNC-polymer interface and the creation of rigid CNC percolating network within the polymer matrix would facilitate an effective stress transfer from the soft polymer matrix to the rigid $\mathrm{CNCs}$, leading to the improvement in the strength and toughness of polymer nanocomposites. Therefore, how to maximally improve the dispersion state, construct strong interfacial adhesion and create percolating network has gained considerable interest in the CNC-reinforced polymer nanocomposite field [16-20]. Consequently, surface modification of CNCs to be compatible or reactive with polymer matrix was inspired. Different surface modification strategies, e.g., ionic interaction [21], urethanization [22], esterification [23], etherification [24], oxidation [25], silylation [26], and polymer grafting through the conventional free radical polymerization [27], ring-opening polymerization [28], atom transfer radical polymerization [29], and thiol-ene "click" reaction [30] have been reported. For example, Ansari et al. [21] hydrophobically modified CNCs using a cationic surfactant - 
dedecyltrimenthylammonium chloride through ionic interaction between the negatively charged sulfate groups on the CNCs and the positively charged ammonium groups on the surfactant. They found that the modified CNCs exhibited superior reinforcement in poly(vinyl acetate) matrix over $\mathrm{CNCs}$ due to the accelerated physical aging process as well as the improved dispersion state and interfacial interaction. Sirvio et al. [25] prepared bifunctional CNCs with aldehyde and carboxyl groups through peroxide and chlorite oxidation. Due to the formation of acetal bonds between the aldehydes of bifunctional CNCs and the hydroxyl groups of poly(vinyl alcohol), up to 2-fold improvement in the tensile strength and Young's modulus was achieved with the addition of $10 \mathrm{wt} \%$ bifunctional CNCs in neat poly(vinyl alcohol) film.

In this article, we reported a novel strategy to reinforce the anionic CMC biopolymer matrix using the cationically modified $\mathrm{CNCs}$ (mCNCs). The mCNCs were synthesized through an alkaline-activated nucleophilic reaction between $\mathrm{CNCs}$ and a cationic surfactant - (2,3epoxypropyl)trimethylammonium chloride (EPTMAC). The successful cationic modification was well validated using several analytic techniques, i.e., Fourier transform infrared spectroscopy (FTIR), solid-state ${ }^{13} \mathrm{C}$ nuclear magnetic resonance (NMR), X-ray photoelectron spectroscopy (XPS), thermogravimetric analyzer (TGA), transmission electron microscope (TEM), zeta potential, and viscosity measurements. Subsequently, mCNC/CMC films with a variable weight ratio of $\mathrm{mCNCs}$ (i.e., 1, 3, 5 and $10 \mathrm{wt} \%$ ) were prepared using the solution casting method. Their processability, mechanical and dynamic mechanical properties, and fracture surface morphology were evaluated and contrasted with those of CNC/CMC films. Distinctive reinforcement phenomena were observed and interpreted in terms of dispersion state and interfacial bonding. 


\section{Experimental section}

\subsection{Materials}

Cellulose nanocrystals (CNCs, BGB Natural ${ }^{\mathrm{TM}} \mathrm{CNC}, 7.4 \mathrm{wt} \%$ suspension, neutral $\mathrm{pH}$, carboxyl content $0.09 \mathrm{mmol} / \mathrm{g}$ ) were purchased from Blue Goose Biorefineries Inc. (Edmonton, AB, CANADA). According to the product information, CNCs were isolated from western hemlock using a transition metal catalyst oxidative method. Carboxymethyl cellulose (CMC, PAC ${ }^{\mathrm{TM}}-\mathrm{L}$, substation degree of carboxylic group > 0.6, white or tan powder, bulk density $40-55 \mathrm{lb} / \mathrm{ft}^{3}$ ) was provided by Hallibuton Company (Houston, TX, USA). Cationic surfactant 2,3-epoxypropyl trimethylammonium chloride (EPTMAC) and sodium hydroxide pellets were purchased from Sigma-Aldrich (St. Louis, MO, USA). All chemicals were used without further purification.

\subsection{Cationic surface modification of CNCs}

The cationic surface modification of CNCs was carried out according to the literatures reported previously with some modifications [24,31]. Two different reaction systems, i.e., diluted and concentrated, were applied to synthesize mCNCs with different substitution degree. In the diluted reaction system, the $7.4 \mathrm{wt} \% \mathrm{CNC}$ suspension was diluted into $1 \mathrm{wt} \%$ using deionized water. In the concentrated system, the $7.4 \mathrm{wt} \% \mathrm{CNC}$ suspension was directly used. The CNC suspension (1 or $7.4 \mathrm{wt} \%$ ) was then activated using sodium hydroxide for $30 \mathrm{~min}$. Subsequently, the EPTMAC was slowly added into the activated CNC suspension. The molar ratio of EPTMAC to anhydroglucose units in CNCs was fixed as 3/1. The cationization was carried out in a round-bottom flask with strongly mechanical stirring for $6 \mathrm{~h}$ at $65^{\circ} \mathrm{C}$. After the cationization, the reaction mixture was diluted using excess deionized water to reduce the alkalinity, and then

poured into a regenerated cellulose dialysis tube (Nominal MWCO 12,000-14,000, Fisher 
Scientific). The tube was dialyzed against excess deionized water in a large water tank for 2 weeks to completely remove unreacted monomers. Finally, the concentrations of $\mathrm{CNC}$ and mCNC suspensions were calculated and then adjusted to $1 \mathrm{wt} \%$ at neutral $\mathrm{pH}$ through proper dilution or evaporation process. The mCNCs prepared from the diluted and concentrated reaction systems were designated as dCNCs and cCNCs, respectively.

\subsection{Preparation of $C N C / C M C$ and $m C N C / C M C$ films}

A series of $\mathrm{CNC} / \mathrm{CMC}$ and $\mathrm{mCNC} / \mathrm{CMC}$ films with different $\mathrm{CNC}$ and $\mathrm{mCNC}$ concentrations (i.e., $0,1,3,5$ and $10 \mathrm{wt} \%$, based on $\mathrm{CMC}$ weight) were prepared using the conventional solution casting method. A constant $25 \mathrm{~g}$ of film-forming suspension (FFS) was prepared for casting each film. In the FFS, the concentration of CMC was fixed as $4 \mathrm{wt} \%$, while the concentration of CNCs or mCNCs varied. In detail, certain amounts of $1 \mathrm{wt} \% \mathrm{CNC}$ or mCNC suspension (i.e., $0,1,3,5$ and $10 \mathrm{~g}$ ) were first mixed with desired amounts of deionized water

(i.e., 24, 23, 21, 19 and $14 \mathrm{~g}$ ) at neutral $\mathrm{pH}$ under vigorous stirring for $1 \mathrm{~h}$. Afterwards, about $1 \mathrm{~g}$ of CMC was carefully added into the resultant suspension. The mixture was vigorously stirred until the CMC was completely dissolved. Then, the FFS was poured onto a glass Petri dish and allowed to dry for five days at room temperature. The films were carefully peeled from the glass Petri dish and have thickness in the range of 80-100 $\mu \mathrm{m}$ depending on the concentration of CNCs and mCNCs. The resultant films were designated as $\mathrm{CNCx} / \mathrm{CMC}, \mathrm{dCNCx} / \mathrm{CMC}$ and $\mathrm{cCNCx} / \mathrm{CMC}$, where $\mathrm{x}$ is the weight percent of $\mathrm{CNCs}$, $\mathrm{dCNCs}$ and $\mathrm{cCNCs}$ relative to $\mathrm{CMC}$.

\subsection{Characterization of CNCs and $m C N C s$}

The $\mathrm{CNC}$ and $\mathrm{mCNC}$ suspensions were dried in an oven under vacuum. The dried films were characterized using FTIR, solid-state ${ }^{13} \mathrm{C}$ NMR, XPS and TGA. FTIR spectra were recorded 
using a Bruker FTIR analyzer (Tensor-27, Bruker Optics Inc., Billerica, MA), equipped with a $\mathrm{Zn} / \mathrm{Se}$ attenuated total reflectance (ATR) crystal accessory, in a transmittance mode with the wavenumber range of $600-4000 \mathrm{~cm}^{-1}$ at a resolution of $4.0 \mathrm{~cm}^{-1}$. Solid-state ${ }^{13} \mathrm{C}$ NMR measurements were performed on a Bruker Avance $400 \mathrm{WB}$ instrument operating at $9.39 \mathrm{~T}$ at room temperature. The magic angle spinning rate was $12 \mathrm{~Hz}$ and a $4 \mathrm{~mm}$ probe was used. The Hartman-Hahn matching procedure was on the base of glycine. The chemical shift was related to adamantane with its low-field peak set to $38.48 \mathrm{ppm}$. XPS spectra were collected on a Specs PHOIBOS-100 spectrometer (SPECS, Berlin, Germany) at $10 \mathrm{kV}$ under a current of $10 \mathrm{~mA}$. Survey spectra were recorded from 600 to $200 \mathrm{eV}$ at pass energy of $40 \mathrm{eV}$ with a scan step of 1.0 $\mathrm{eV}$. High-resolution spectra were recorded at pass energy of $40 \mathrm{eV}$ with a scan step of $0.1 \mathrm{eV}$. The mass percentage of elements was calculated using SpecLab software. The high-resolution C 1s spectra were resolved into different contribution of bonded carbon using a nonlinear leastsquare curve-fitting program (XPSPEAK41 software). The spectra were calibrated by setting the C-C (C-H) contribution in the $\mathrm{C} 1 \mathrm{~s}$ emission at $285.0 \mathrm{eV}$ [32]. The morphology of CNCs and mCNCs was observed using a Transmission Electron Microscope (TEM, JEM 1400, JEOL) at an accelerating voltage of $120 \mathrm{kV}$. For the sample preparation, $1 \mathrm{wt} \% \mathrm{CNC}$ and $\mathrm{mCNC}$ suspensions were further diluted to $0.02 \mathrm{wt} \%$ with deionized water. To facilitate the deposition of the hydrophilic nanoparticles, the copper grids (CF-400-CU, Electron Microscopy Sciences, Hatfield, PA, USA) were treated by glow discharge. Afterwards, a droplet of $0.02 \mathrm{wt} \% \mathrm{CNC}$ and $\mathrm{mCNC}$ suspension was placed onto the glow-discharged copper grid, followed by rinsing throughout with $2 \mathrm{wt} \%$ uranyl acetate solution for 2 min to stain the CNCs and mCNCs for a better observation. The zeta potential values of $0.1 \mathrm{wt} \% \mathrm{CNC}$ and $\mathrm{mCNC}$ suspensions at neutral $\mathrm{pH}$ were determined using a ZetaTrac analyzer (MicroTrac Inc., Largo, FL, U.S.A.). Five replicates 
were performed for each sample and the average value was calculated. The steady-state viscosity of $1 \mathrm{wt} \% \mathrm{CNC}$ and $\mathrm{mCNC}$ suspensions was measured using a stress-controlled rheometer (AR 2000, TA Instrument Inc., New Castle, DE, USA) with a cone-and-plate geometry (cone angle: 20; diameter: $40 \mathrm{~mm}$; truncation: $56 \mu \mathrm{m}$ ) in the shear range from 1 to $1000 \mathrm{~s}^{-1}$ at $25^{\circ} \mathrm{C}$.

\subsection{Characterization of $m C N C / C M C$ FFS and dried films}

Steady-state viscosity of FFS was measured using the AR 2000 rheometer with the same geometry and shear range as mentioned previously. Mechanical properties of films were also measured using the AR 2000 rheometer with a solid clamp in tension mode. A constant strain rate of $10 \mu \mathrm{m} / \mathrm{s}$ was used. Before the tensile tests, the films were conditioned in desiccators to reach equilibrium at a relative humidity $(\mathrm{RH})$ value of $55 \%$ over $\mathrm{Mg}\left(\mathrm{NO}_{3}\right)_{2}$ saturated solution; and then cut into dumbbell shape according to the ASTM standard D638-V. The thickness of each specimen was tested using a Mitutoyo digimatic indicator. The Young's modulus was calculated from the slope of the initial linear region of the stress/strain curves. Five replicates were collected and the average values were reported. Dynamic mechanical properties of films were conducted using a TA Q800 dynamic mechanical analyzer (DMA, TA Instrument Inc., New Castle, DE, USA) equipped with a film tension clamp. The frequency sweep was performed over a frequency range from 1 to $100 \mathrm{~Hz}$ at a fixed strain of $0.1 \%$ at $35^{\circ} \mathrm{C}$. The frequency was exponentially increased at a rate of 5 data points per decade. The specimen was in rectangular shape (approximate $13 \mathrm{~mm} \times 3.18 \mathrm{~mm}$ ). Fracture surface morphology of films from tensile tests were observed using a field emission scanning electron microscopy (FE-SEM, a FEI QuantaTM 3D FEG dual beam SEM/FIB system, Hillsboro, OR) at an accelerating voltage of $5 \mathrm{kV}$. Before observation, each sample was mounted on an aluminum stub and coated with a layer of gold using a sputter coater. 


\section{Results and discussion}

\subsection{Synthesis and Characterization of $m C N C s$}
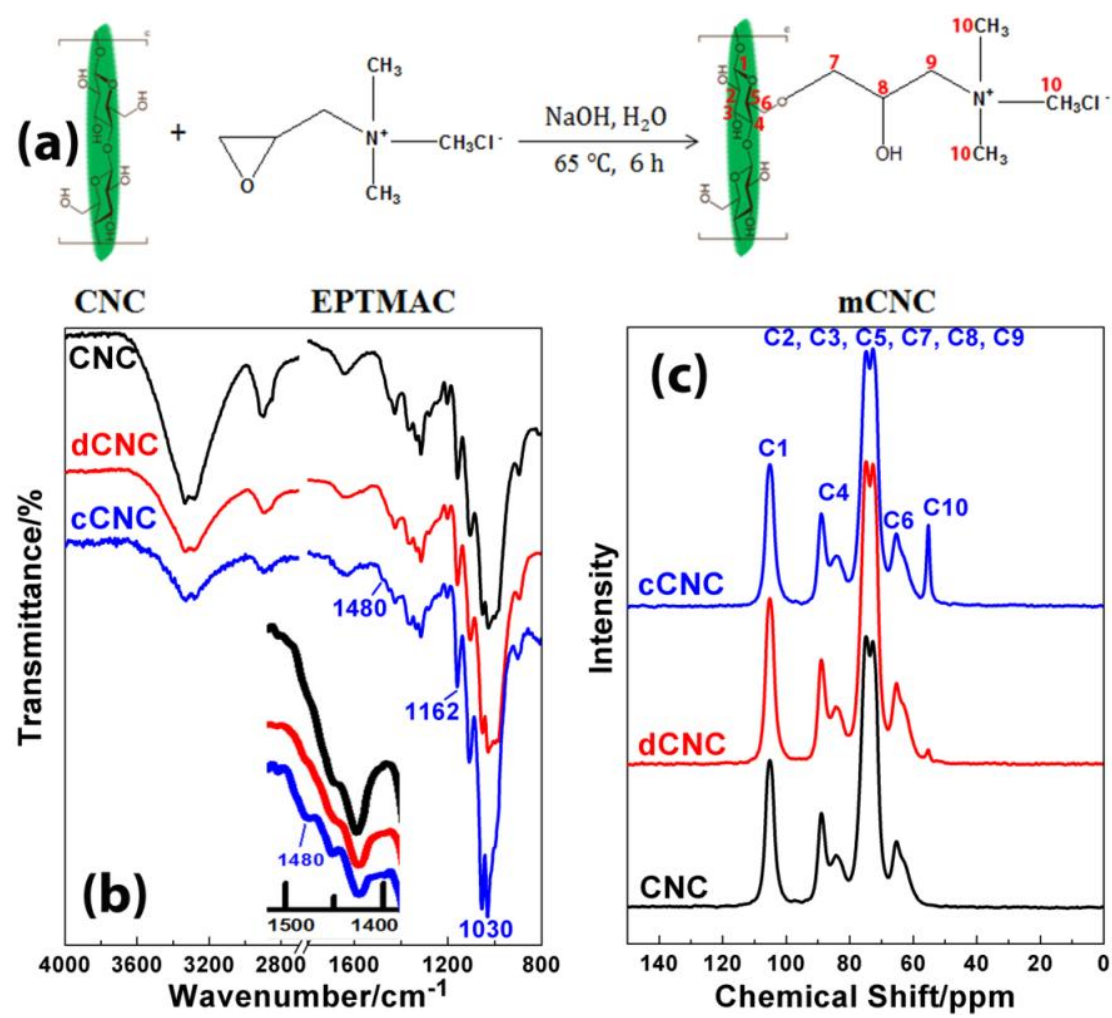

Figure 1. Synthesis and chemical structure of CNCs and mCNCs: (a) synthetic scheme for cationic surface functionization of CNCs with EPTMAC; (b) and (c) FTIR and solid-state ${ }^{13} \mathrm{C}$ NMR spectra of CNCs and mCNCs, respectively.

Cationic surface modification of CNCs with EPTMAC underwent a nucleophilic reaction between the alkali-activated hydroxyl groups of CNCs and the epoxide groups of EPTMAC, as illustrated in Figure 1a. As known, the epoxide group is cyclic ether, consisting of one oxygen atom linked to two carbon atoms. Due to the high polarity of these two $\mathrm{C}-\mathrm{O}$ bonds, the ring of cyclic ether is greatly strained. The carbons in the epoxide group are therefore highly reactive electrophiles, especially with the presence of strong nucleophiles. Meanwhile, through the pre- 
treatment of $\mathrm{CNCs}$ using a strong alkaline solution, i.e., $\mathrm{NaOH}$, the hydroxyl groups on the $\mathrm{CNC}$ backbone were activated, which could act as reactive nucleophiles. Therefore, with the addition of EPTMAC monomer into the alkali-activated CNC suspension, a nucleophilic reaction took place by an $\mathrm{S}_{\mathrm{N}} 2$ mechanism, i.e., the nucleophilic attack occurred in the less substituted carbon. It is worth noting that during the cationization process, side reactions, i.e., hydrolysis of EPTMAC occurred as well, which would yield an adverse influence on the substitution degree of mCNCs [31]. One of the most critical parameters affecting the hydrolysis of EPTMAC is the water content of reaction system. In general, a more aggressive hydrolysis took place in the reaction system with higher water content. Therefore, the substitution degree of mCNCs could be tailored by controlling the water content of reaction system. In the present study, two reaction systems with different water contents, i.e., concentrated and diluted, were applied for the cationic surface modification of CNCs. It is expected that the cCNCs would have much higher substitution degree than dCNCs.

Successful surface cationization of CNCs was confirmed by several analytical techniques, including FTIR, solid-state ${ }^{13} \mathrm{C}$ NMR and XPS. Figure $1 \mathrm{~b}$ shows the FTIR spectra of neat CNCs and mCNCs. CNCs exhibited strongly characteristic absorption peaks of cellulose, i.e., O-H stretching vibration between 3000 and $3700 \mathrm{~cm}^{-1}, \mathrm{CH}_{2}$ asymmetric stretching vibration at 2920 $\mathrm{cm}^{-1}$, C-H stretching vibration at $2900 \mathrm{~cm}^{-1}, \mathrm{CH}_{2}$ symmetric stretching vibration at $2850 \mathrm{~cm}^{-1}$, O$\mathrm{H}$ bending of absorbed moisture at $1640 \mathrm{~cm}^{-1}, \mathrm{OCH}$ and $\mathrm{HCH}$ in-plane bending vibrations at $1427 \mathrm{~cm}^{-1}, \mathrm{CH}$ deformation vibration at $1367 \mathrm{~cm}^{-1}$, C-O-C asymmetric stretching vibration at $1160 \mathrm{~cm}^{-1}$, glucose ring stretching vibration at $1108 \mathrm{~cm}^{-1}$, and C-O stretching vibration at 1030 $\mathrm{cm}^{-1}$. In comparison with $\mathrm{CNCs}$, mCNCs displayed much weaker $\mathrm{O}-\mathrm{H}$ stretching vibration peaks between 3000 and $3700 \mathrm{~cm}^{-1}$, but much stronger C-O-C asymmetric stretching vibration peak at 
$1160 \mathrm{~cm}^{-1}$ and $\mathrm{C}-\mathrm{O}$ stretching vibration peak at $1030 \mathrm{~cm}^{-1}$, attributing to the consumption of hydroxyl groups and the formation of new ether groups during the surface cationization. Furthermore, a new peak corresponding to the $\mathrm{CH}_{2}$ bending mode and $\mathrm{CH}_{3}$ groups of EPTMAC substituents appeared at $1480 \mathrm{~cm}^{-1}$ after surface cationization, especially for cCNCs (inserted picture in Figure 1b).

Solid-state ${ }^{13} \mathrm{C}$ NMR spectra provided more convinced evidence on the successful surface cationization, as depicted in Figure 1c. CNCs showed typical resonance peaks of cellulose type I at 105.2 (C1), 88.9 (crystalline C4), 84.3 (amorphous C4), 74.8, 72.7 (C2, C3 and C5), and 65.3 (C6) ppm. After surface cationization, a new resonance signal at $55.2 \mathrm{ppm}$ corresponding to the methyl carbons of the EPTMAC substituents (C10) appeared. Moreover, it was observed that cCNCs exhibited much more intensive signal at $55.2 \mathrm{ppm}$ over dCNCs, demonstrating the introduction of more EPTMAC substituents on the surface of cCNCs. It was reported that the crystallinity index (CrI) can be calculated using the following equation [33]:

$$
\operatorname{CrI}=I_{c 4, c r y} /\left(I_{c 4, c r y}+I_{c 4, a m}\right)
$$

where $\mathrm{I}_{\mathrm{C} 4 \text {,cry }}$ and $\mathrm{I}_{\mathrm{C} 4 \text {,am }}$ are integrals of the resonance signals for $\mathrm{C} 4$ at $88.9 \mathrm{ppm}$ and $\mathrm{C} 4$ at 84.3 ppm, respectively. CNCs, dCNCs and cCNCs had crystalline index of $0.561,0.565$ and 0.558 , respectively. The CrI values were found to be almost unchanged, revealing that cationic modification did not alter the crystalline structure of CNCs. Thus, the cationic modification mainly occurred on the surface of CNCs. 

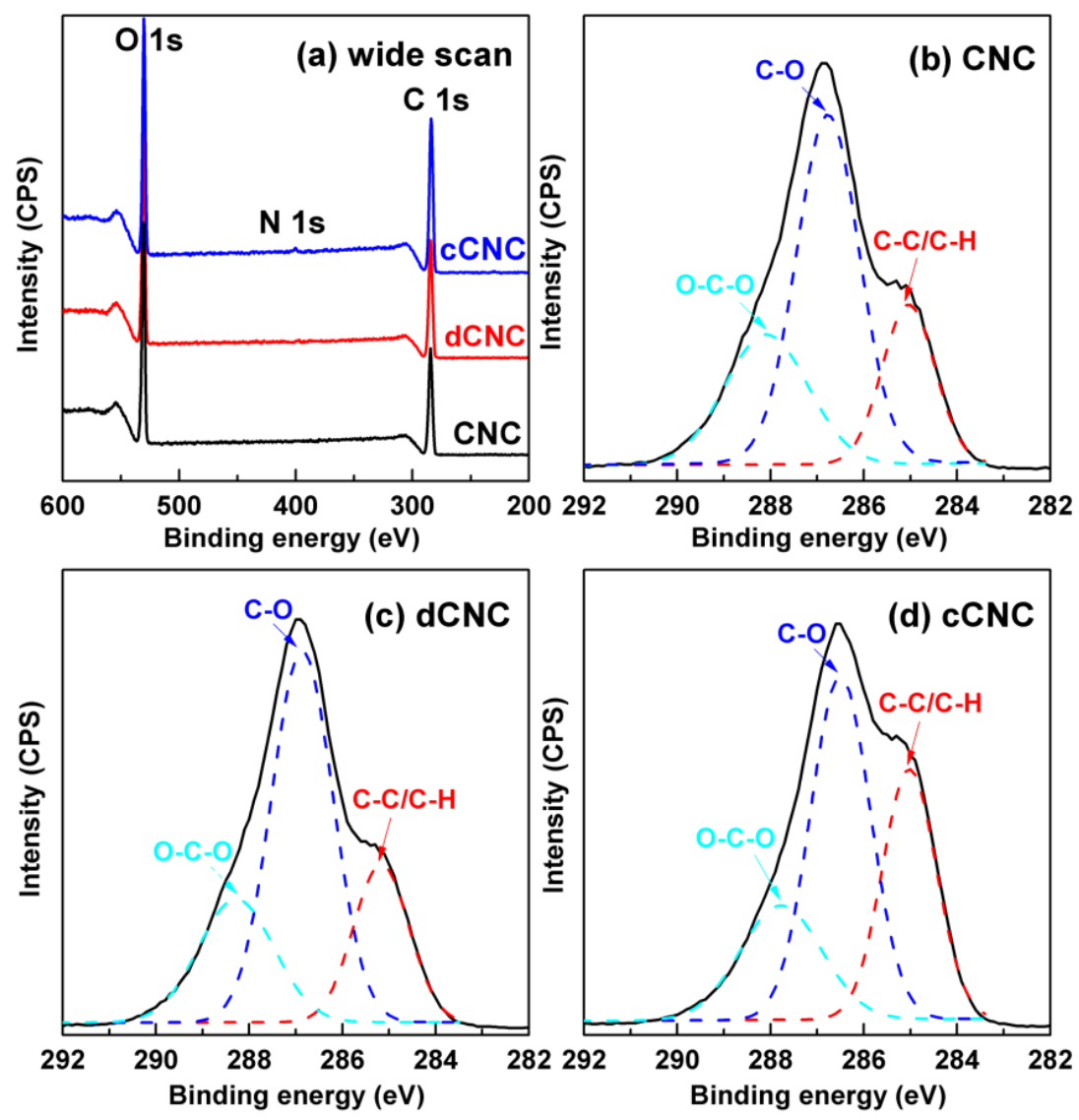

Figure 2. XPS Spectra of CNCs and mCNCs: (a) wide scan; and deconvolution of the high resolution $\mathrm{C}$ 1s peak for (b) CNCs, (c) dCNCs and (d) cCNCs.

XPS has the advantage of determining the chemical composition of a surface layer with a thickness of approximately 3-6 nm [34]. Considering the cationic modification primarily took place on the surface of CNCs, XPS is a more powerful tool to examine the change in the composition and chemical structure of CNCs before and after cationic modification. The wide scan XPS spectra of CNCs and mCNCs are presented in Figure 2a. As shown, only C and O elements were detected in $\mathrm{CNCs}$ within the investigated range. After cationic modification, in addition to $\mathrm{C}$ and $\mathrm{O}$ elements, $\mathrm{N}$ element resulting from the ammonium groups of EPTMAC substituents appeared as well. The atomic surface composition of CNCs and mCNCs was further calculated, as summarized in Table $\mathrm{S} 1$. The $\mathrm{O} / \mathrm{C}$ ratio for $\mathrm{CNCs}$ was found to be 0.56 , which was 
lower than the theoretical $\mathrm{O} / \mathrm{C}$ ratio of neat cellulose (0.83), presumably due to the presence of a few amounts of contaminants on the surface, i.e., non-cellulosic residues from wood resources and adsorbed molecules because of air exposure [35]. The successful cationic modification was well confirmed through the increase in the mass percentage of both $\mathrm{C}$ and $\mathrm{N}$ as well as the decrease in the mass percentage of $\mathrm{O}$ element and $\mathrm{O} / \mathrm{C}$ ratio in mCNCs. Moreover, in comparison with dCNCs, cCNCs had higher mass percentage of $\mathrm{C}$ and $\mathrm{N}$, indicating that the surface of cCNCs was richer in $\mathrm{C}$ and $\mathrm{N}$ due to the introduction of more EPTMAC substituents, again. From the XPS spectra results, the substitution degree values for mCNCs were determined. The calculated substitution degree values for dCNCs and cCNCs were 0.05 and 0.12 , respectively (see supplementary data). As expected, with decrease in the water content from 99 to $92.7 \mathrm{wt} \%$ in the reaction system, the substitution degree increased by approximately 2.4 fold.

The deconvolution of the high resolution $\mathrm{C}$ 1s peak of CNCs and mCNCs were also conducted, giving a further insight into the change in the structure of bonded carbon before and after surface cationization. As shown in Figures 2b-2c, the high-resolution C 1s peaks of CNCs and mCNCs were resolved into three Gaussian component peaks, i.e., C-C or C-H at $285 \mathrm{eV}, \mathrm{C}-\mathrm{O}$ at $286.5 \mathrm{eV}$ and $\mathrm{O}-\mathrm{C}-\mathrm{O}$ at $288 \mathrm{eV}$ [32]. It is worth noting that it was difficult to derive the $-\mathrm{O}-\mathrm{C}=\mathrm{O}$ peak at $289 \mathrm{eV}$ through deconvolution, which might be due to the fact that the amount of carboxyl group in CNCs was tiny $(0.09 \mathrm{mmol} / \mathrm{g})$ according to the product information. The percentage of differently bonded carbon atoms from C 1s high-resolution XPS spectra is also summarized in Table S1. Due to the introduction of long alkane chains in EPTMAC substituents, substantial increase in the percentage of C-C or C-H was observed for mCNCs, especially for cCNCs. FTIR, solid-state ${ }^{13} \mathrm{C}$ NMR and XPS results strongly confirmed that cationic surfactant EPTMAC was successfully grafted onto the surface of CNCs through an alkali-activated etherification reaction 
and the substitution degree of mCNCs was well tailored by controlling the water content of reaction system.

Next, the effect of surface cationization on the thermal stability, morphology, dispersion state and rheological properties of CNCs was investigated. TGA and corresponding differential thermogravimetric analysis (DTG) curves of CNCs and mCNCs are depicted in Figures S1a and $\mathrm{S} 1 \mathrm{~b}$, respectively. In general, the thermal decomposition of neat cellulose consisted of three main steps, i.e., the evaporation of moisture at low temperature range, the degradation of primary glycosidic units to char residues at intermediate temperature range, and the further oxidation and breakdown of the char residues at high temperature range [36]. In case of CNCs, in addition to these three typical decomposition steps, a new thermal decomposition step occurring from 150 to $250{ }^{\circ} \mathrm{C}$ was seen, presumably due to the rapid pyrolysis of glycosylic units catalyzed by carboxyl groups on the surface of CNCs. Similar thermal decomposition phenomena were also observed for TEMPO-oxidized cellulose nanofibers and sulfuric acid-hydrolyzed CNCs because of the presence of carboxyl and sulfate groups on the backbone, respectively $[37,38]$. mCNCs exhibited different thermal decomposition patterns compared to CNCs. In particular, with increase in the substitution degree, the TGA curves shifted toward higher temperatures, indicating the improved thermal stability. Furthermore, the decomposition step ranging from 150 to $250{ }^{\circ} \mathrm{C}$ was found to disappear gradually. Two possible origins accounted for these phenomena. First, the pretreatment using $\mathrm{NaOH}$ neutralized the carboxyl groups, and thus largely eliminated the adverse influence of carboxyl groups on the thermal stability. Second, the EMPTAC substituents covered the surface of CNCs, which could act as a barrier to slow down the degradation rate. The improved thermal stability was also verified from DTG curves. As shown in Figure S1b, the maximum decomposition temperature $\left(\mathrm{T}_{\max }\right)$ shifted from $313.6{ }^{\circ} \mathrm{C}$ for $\mathrm{CNCs}$ to 323.0 and $326.6{ }^{\circ} \mathrm{C}$ 
for dCNCs and cCNCs, respectively. Surface cationization led to approximate $10^{\circ} \mathrm{C}$ increase in the $\mathrm{T}_{\max }$ of CNCs, whereas only $3.6{ }^{\circ} \mathrm{C}$ increase in the $\mathrm{T}_{\max }$ was achieved with increase in the substitution degree of mCNCs from 0.05 to 0.12 , suggesting that the influence of elimination of carboxyl groups on the thermal stability was more significant compared with barrier effect of EMPTAC substituents.

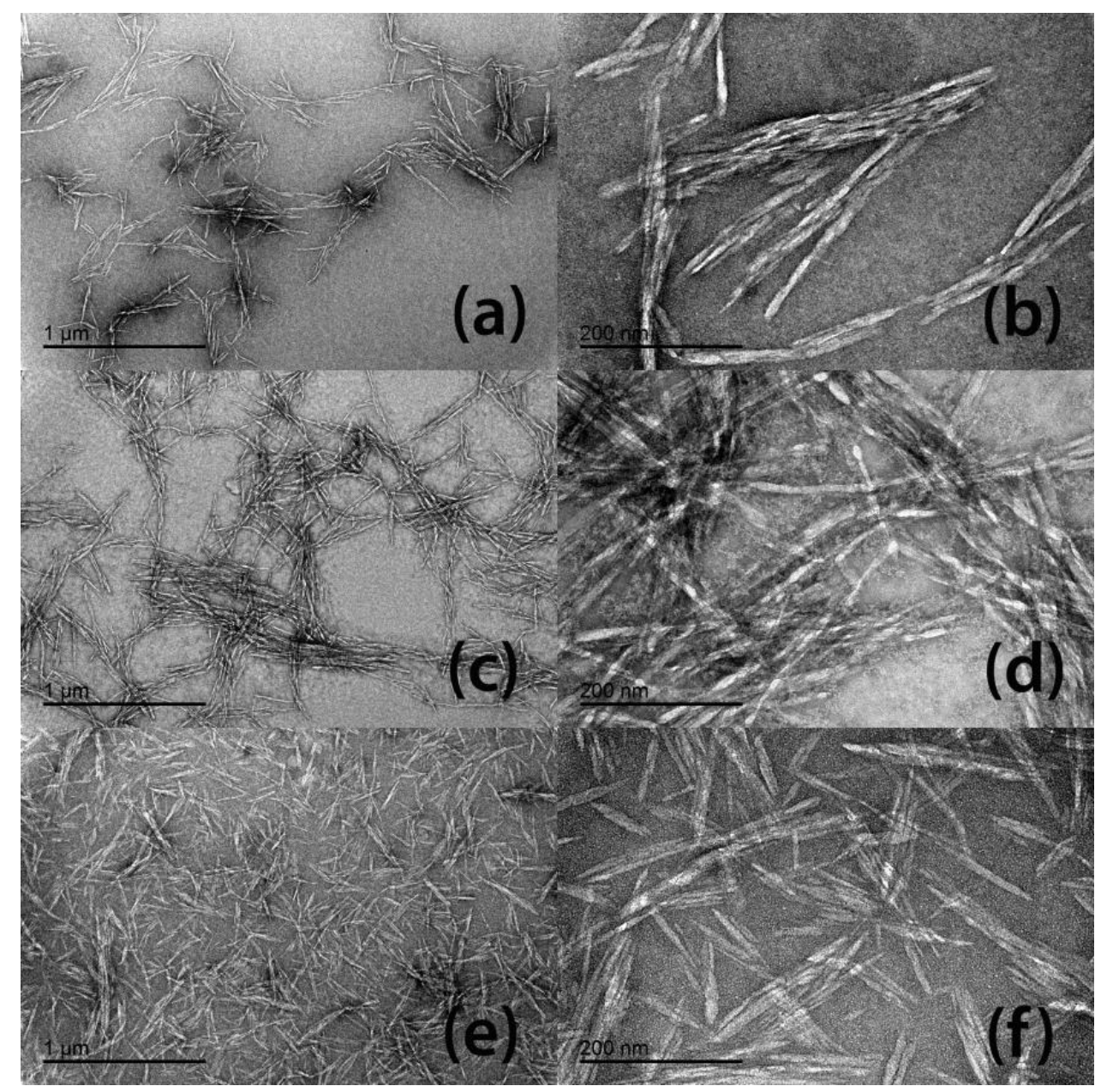

Figure 3. TEM micrographs of CNCs and mCNCs: (a) and (b) CNCs; (c) and (d) dCNCs; and (e) and (f) cCNCs. Scale bar: (a), (c) and (e) $1 \mu \mathrm{m}$; and (b), (d) and (f) $200 \mathrm{~nm}$.

Surface cationization also caused the distinctive morphology and dispersion state of CNCs and mCNCs in aqueous solution, as shown in Figure 3. Individual $\mathrm{CNC}$ exhibited rod-like 
morphology with 100-200 $\mathrm{nm}$ in length and 5-20 nm in width. Due to the occurrence of strong hydrogen bonding among $\mathrm{CNCs}$, several $\mathrm{CNCs}$ aggregated to form longitudinally oriented bundles (Figures $3 \mathrm{a}$ and $3 \mathrm{~b}$ ). The formation of such bundles or aggregates largely reduced the surface area of CNCs and thus hindered their reinforcing ability, which was one of the reasons why the cationic surface modification was carried out in this study. It is expected that after surface cationization, mCNCs would carry more charges, i.e., quaternary ammonium cations on the surface than CNCs with tiny carboxyl groups $(0.09 \mathrm{mmol} / \mathrm{g})$, leading to the formation of homogeneous dispersion state of $\mathrm{mCNCs}$ in aqueous solution through electrostatic repulsion forces. However, by comparing the TEM micrographs of dCNCs (Figures 3c and 3d) with cCNCs (Figures 3e and 3f), it was found that the substitution degree played a key role. At a low substitution degree, dCNCs still aggregated and appeared as bundles. These bundles seem to be further randomly attached, creating larger aggregates over CNCs. In contrast to dCNCs, cCNCs showed very uniform dispersion state in an aqueous solution. For charged nanoparticles, their dispersion state was directly associated with their surface interaction between adjacent nanoparticles. In case of $\mathrm{mCNC}$ dispersed in aqueous solution, different surface interactions, including attraction force (e.g., hydrogen bonding between hydroxyl groups and hydroxyl/quaternary ammonium groups) and repulsion force (e.g., electrostatic repulsion between two quaternary ammonium cations) could be present. It is believed that these two opposite actions would compete with each other, resulting in the distinctive dispersion state of mCNCs in aqueous solution depending on the substitution degree of mCNCs. Therefore, it is speculated that the uniform dispersion of cCNCs in aqueous solution was ascribed to the formation of profound repulsion force over attraction force; whereas the poor dispersion state of dCNCs in aqueous solution was most likely due to the occurrence of predominant attraction 
force over repulsion force. In order to confirm these assumptions, their zeta potential values were measured. It was found that $0.1 \mathrm{wt} \% \mathrm{CNC}, \mathrm{dCNC}$ and $\mathrm{cCNC}$ suspensions at neutral $\mathrm{pH}$ had zeta potential values of $-25.01 \pm 1.73,+17.54 \pm 1.40$ and $+32.47 \pm 2.47 \mathrm{mV}$, respectively. Compared with dCNC suspension, cCNC suspension exhibited much higher zeta potential value due to the presence of more EPTMAC substituents. Such higher zeta potential value allowed cCNCs to repel each other through electrostatic repulsion forces more effectively, leading to the observed better dispersion state. However, it is worth noting that the absolute zeta potential value of dCNC suspension was even lower than that of CNC suspension, resulting in the formation of even larger aggregates over CNCs.

Assuming that the above mentioned attraction and repulsion forces co-existed in the $\mathrm{mCNC}$ suspension, their balance would be well reflected in the steady-state viscosity and shear stress. Therefore, the steady-state shear viscosity and shear stress of $1 \mathrm{wt} \% \mathrm{CNC}$ and $\mathrm{mCNC}$ suspensions were investigated as a function of shear rate, as shown in Figure 4a. As expected, at a fixed shear rate, both the viscosity and shear stress increased in the order of $\mathrm{cCNCs}<\mathrm{CNCs}<$ dCNCs. These results were fully consistent with the TEM observations, strongly confirming the above assumption that the repulsion force was predominant over attraction force in the $\mathrm{cCNC}$ suspension; whereas the attraction force was predominant over repulsion force in the $\mathrm{dCNC}$ suspension. Overall, through the above TGA, TEM and viscosity investigations, it can be concluded that in comparison with $\mathrm{CNCs}$ and dCNCs, cCNCs possessed several advantages, i.e., higher substitution degree, better thermal stability, more uniform dispersion state and lower viscosity. It is expected that these advantages would make cCNCs more ideal for CMC biopolymer reinforcement. 


\subsection{Processability and viscosity of $m C N C / C M C F F S$}
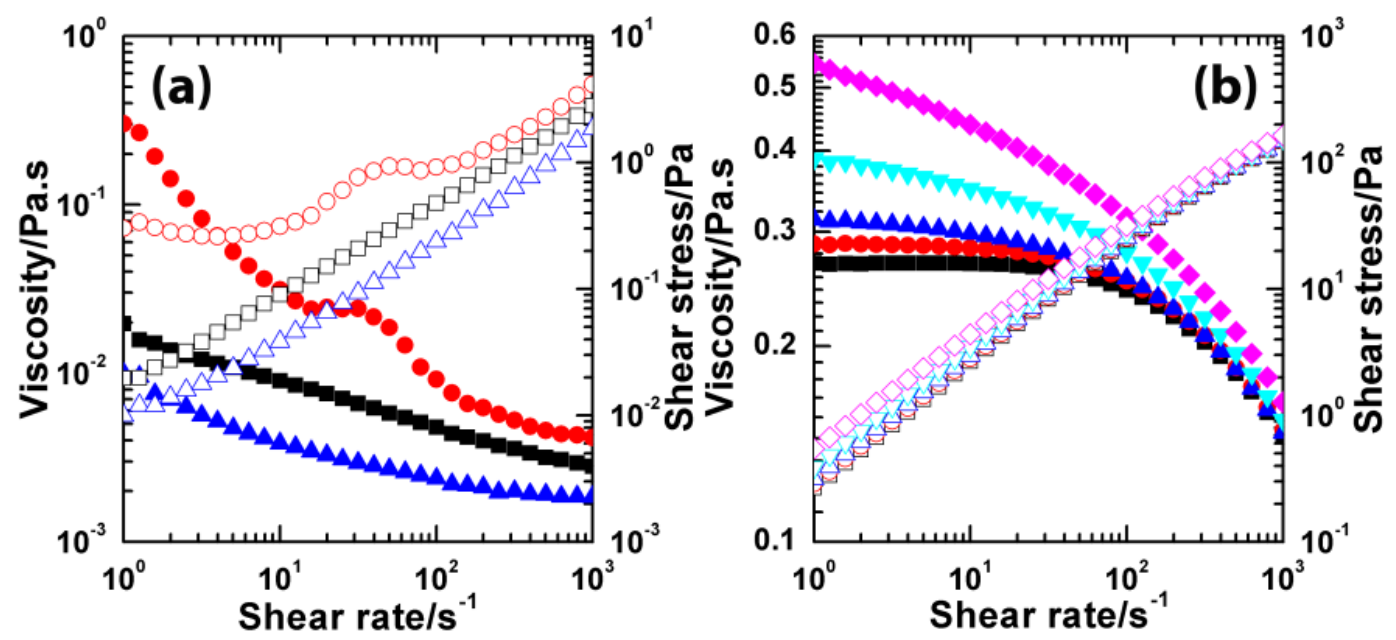

Figure 4. Steady-state shear viscosity (solid symbols) and shear stress (open symbols) versus shear rate of (a) $1 \mathrm{wt} \%$ suspension of CNCs (black squares), dCNCs (red circles) and cCNCs (blue triangles); and (b) cCNC/CMC FFS with 0 (black squares), 1 (red circles), 3 (blue up triangles), 5 (cyan down triangles), and 10 (Magenta diamonds) wt\% cCNCs.

Afterward, the $\mathrm{cCNC} / \mathrm{CMC}$ FFS with a variable weight ratio of cCNCs from 1 to $10 \mathrm{wt} \%$ were prepared by mixing $\mathrm{CMC}$ with $\mathrm{cCNC}$ suspension. It was found that when the viscosity of FFS was too high, it was very difficult to eliminate air bubbles as well as to achieve the uniform dispersion state. Furthermore, the resultant films were rough, inhomogeneous, less transparent and even difficult to peel and handle. It is therefore very essential to study the processability of FFS in terms of viscosity in order to obtain high-quality films. Figure $4 b$ shows the steady-state shear viscosity and shear stress as a function of shear rate for cCNC/CMC FFS with different cCNC concentrations. The neat CMC FFS exhibited a Newtonian behavior in the shear region from 1 to $20 \mathrm{~s}^{-1}$ and a shear-thinning behavior in the shear region from 20 to $1000 \mathrm{~s}^{-1}$. The CMC FFS contained looping and highly entangled CMC molecular chains, which created high resistance to flow at the low shear rate region. As the shear rate continuously increased, these 
entangled $\mathrm{CMC}$ molecular chains were gradually aligned to the shear direction, leading to the reduction in the viscosity [39]. The addition of cCNCs gradually changed the viscosity pattern of CMC FFS. At low cCNC concentrations (e.g., 1 and 3 wt $\%$ ), cCNC/CMC FFS exhibited similar viscosity curve with $\mathrm{CMC} / \mathrm{FFS}$, indicating the looping and entangled CMC network still dominated; whereas beyond $5 \mathrm{wt} \%$, the Newtonian region disappeared and a more significant shear-thinning behavior was observed in the full studied region. This transition was mostly related to the formation of percolation network within the CMC solution [12].

The Carreau-Yasuda model is usually used to describe the pseudoplastic fluid with an asymptotic shear viscosity at zero, which has the following form [40]:

$$
\eta=\eta_{0}\left[1+(\lambda \dot{\gamma})^{\alpha}\right]^{\frac{n-1}{\alpha}}
$$

where $\eta$ is the shear viscosity, $\eta_{0}$ is the zero shear viscosity, $\lambda$ is a time constant, $\dot{\gamma}$ is the shear rate, $\alpha$ is the dimensionless parameter, and $\mathrm{n}$ is the power law exponent. The derived rheological parameters for cCNC/CMC FFS with different cCNC concentrations are summarized in Table S2. The zero shear viscosity values are $0.269,0.288,0.317,0.412$ and $0.690 \mathrm{~Pa}$. s for cCNC/CMC FFS with $0,1,3,5$ and $10 \mathrm{wt} \%$ cCNCs, respectively. Recently, Miri et al [12] investigated the influence of CNC concentration on the zero shear viscosity of CMC/starch/CNC FFS. They found that the zero shear viscosity values were approximately $0.25,0.38,0.90$ and $2.75 \mathrm{~Pa} . \mathrm{s}$ for $\mathrm{CMC} / \mathrm{starch} / \mathrm{CNC}$ FFS with 0, 0.5, 2.5 and $5.0 \mathrm{wt} \%$ of CNCs, respectively; and demonstrated that such viscosity values were suitable for the film casting process. The zero shear viscosity values of the present cCNC/CMC FFS were much lower than those observed by Miri et al [12]; accordingly, highly smooth, uniform, transparent and easily peeled cCNC/CMC films were produced (Figure S2). 


\subsection{Mechanical properties of $m C N C / C M C$ films}
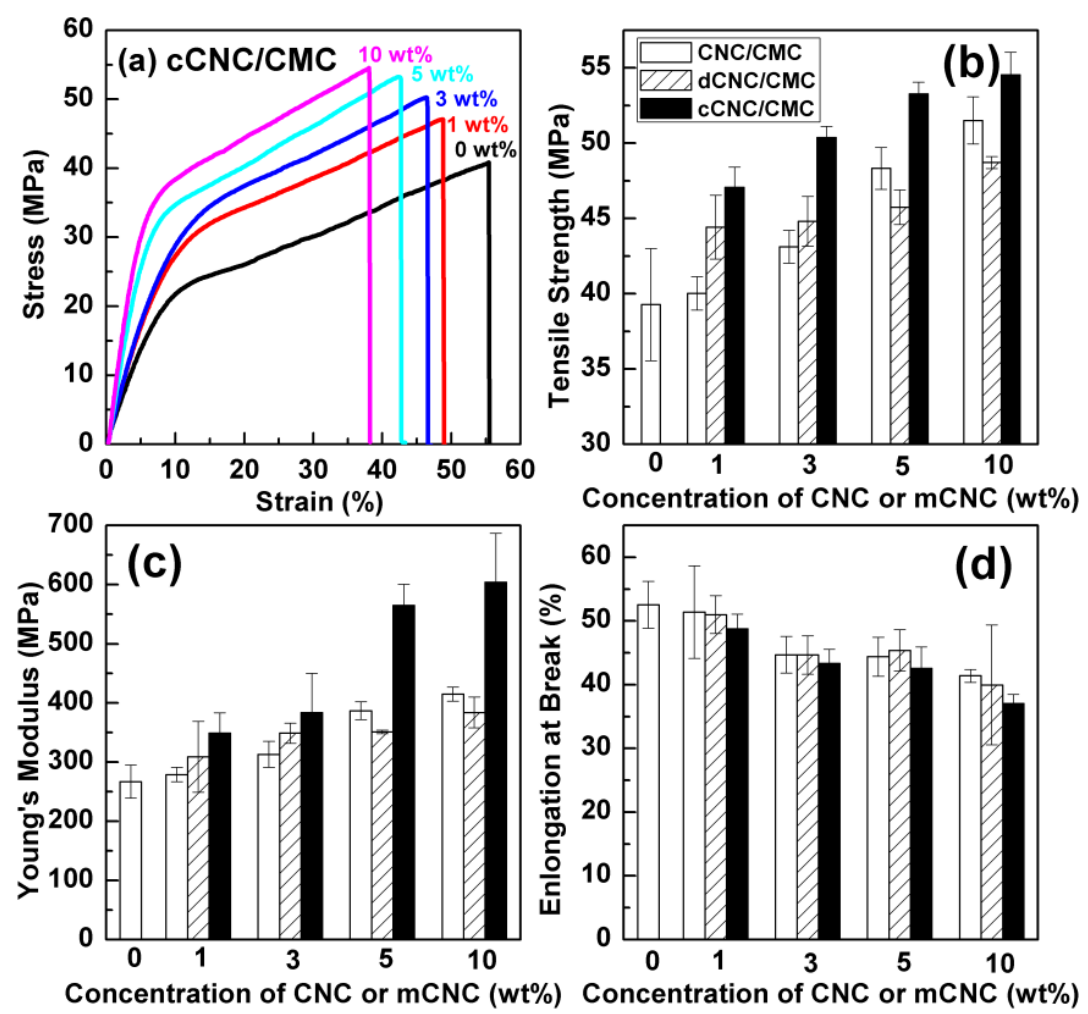

Figure 5. Mechanical properties of $\mathrm{CNC} / \mathrm{CMC}$ and $\mathrm{mCNC} / \mathrm{CMC}$ films: (a) Typical stress-strain curves for $\mathrm{cCNC} / \mathrm{CMC}$ films with different $\mathrm{cCNC}$ concentrations; and comparative study on the mechanical properties of $\mathrm{CNC} / \mathrm{CMC}, \mathrm{dCNC} / \mathrm{CMC}$ and $\mathrm{cCNC} / \mathrm{CMC}$ films: (a) tensile strength, (b) Young's modulus and (c) elongation.

The representative stress-strain curves for $\mathrm{cCNC} / \mathrm{CMC}$ films with different $\mathrm{cCNC}$ concentrations are presented in Figure 5a. Neat CMC film exhibited a tensile strength of 39.27 MPa, a Young's modulus of $219 \mathrm{MPa}$ and an elongation at break of 52.57\%. Compared with the previously reported results [7-13], the present CMC film had lower Young's modulus but higher elongation. Such discrepancies might be ascribed to the differences in the molecular weight and substitution degree of CMC as well as the tensile testing conditions, e.g., the RH value and strain speed. As expected, cCNCs exhibited noticeable reinforcement in CMC films. Both tensile strength and 
Young's modulus of cCNC/CMC films were gradually improved with increase in the $\mathrm{cCNC}$ concentration from 1 to $10 \mathrm{wt} \%$. When $10 \mathrm{wt} \% \mathrm{cCNCs}$ were incorporated, the maximum tensile strength (54.53 MPa) and Young's modulus (375 MPa) were achieved, which were 1.39 and 1.72 times of neat CMC film. However, the elongation at break was found to be continuously decreased. The lowest elongation (37.07\%) was obtained, when $10 \mathrm{wt} \%$ cCNCs were added as well. Recently, Son et al [7] used the reduced graphene oxide ( $\mathrm{rGO}$ ) to reinforce CMC matrix. They observed that when $2 \mathrm{wt} \% \mathrm{rGO}$ was added, the film was quite brittle with a low elongation of $2.03 \%$. The present $\mathrm{cCNC} / \mathrm{CMC}$ film had much larger elongation value than $\mathrm{rGO} / \mathrm{CMC}$ film, demonstrating the better toughness, superior processability and wider applicability of cCNC/CMC film. In an attempt to determine the effectiveness of surface cationization of CNCs on the CMC matrix reinforcement, comparative studies on the mechanical properties of $\mathrm{CNC} / \mathrm{CMC}$ and $\mathrm{mCNC} / \mathrm{CMC}$ films were also carried out, as shown in Figures 5b-5d. It is clear that the $\mathrm{cCNC} / \mathrm{CMC}$ films possessed superior mechanical performance over both $\mathrm{CNC} / \mathrm{CMC}$ and $\mathrm{dCNC} / \mathrm{CMC}$ films at any concentrations. For example, at a concentration of $5 \mathrm{wt} \%$, the $\mathrm{cCNC} / \mathrm{CMC}$ film had the tensile strength of $53.26 \mathrm{MPa}$ and Young's modulus of $358 \mathrm{MPa}$, which were 4.94 and $57 \mathrm{MPa}$ higher than those of $\mathrm{CNC} / \mathrm{CMC}$ film, respectively. However, it was very surprisingly observed that at high concentrations (e.g., 5 and $10 \mathrm{wt} \%$ ), the $\mathrm{dCNC} / \mathrm{CMC}$ films even had lower tensile strength and Young's modulus than $\mathrm{CNC} / \mathrm{CMC}$ films. These phenomena strongly demonstrated that the reinforcing capacity of mCNCs in CMC matrix was significantly governed by their substitution degree. 

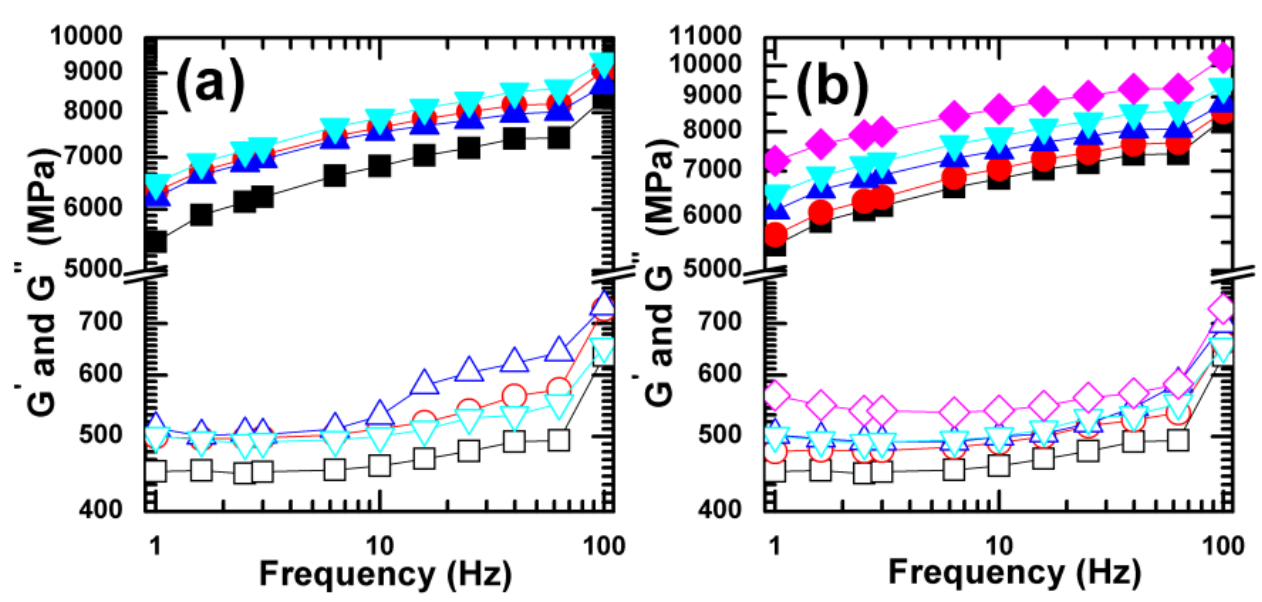

Figure 6 Storage modulus $G^{\prime}$ (solid symbols) and loss modulus $G^{\prime \prime}$ (open symbols) versus frequency of (a) neat CMC (black squares), CNC5/CMC (red circles), dCNC5/CMC (blue up triangles) and cCNC5/CMC (cyan down triangles) films; and (b) cCNC/CMC films with with 0 (black squares), 1 (red circles), 3 (blue up triangles), 5 (cyan down triangles), and 10 (Magenta diamonds) wt $\%$ cCNCs.

The superior reinforcing capacity of cCNCs in CMC matrix was further confirmed by the dynamic frequency sweep tests. As shown in Figure 6a, the presence of CNCs, dCNCs and cCNCs substantially enhanced both the storage and loss moduli of CMC matrix. The storage modulus of films increased in an order of dCNC5/CMC $<$ CNC5/CMC $<$ cCNC5/CMC. On the contrary, the loss modulus of films increased by an opposite order of cCNC5/CMC < CNC5/CMC < dCNC5/CMC. These observations indicated the higher stiffness of cCNC5/CMC than $\mathrm{CNC} 5 / \mathrm{CMC}$ and $\mathrm{dCNC} 5 / \mathrm{CMC}$ films, which were in a well agreement with tensile results. Figure $6 \mathrm{~b}$ shows the influence of $\mathrm{cCNC}$ concentration on the viscoelastic properties of cCNC/CMC films. It was observed that the storage modulus gradually increased with increase in cCNC concentration, showing the effectiveness of cCNCs in CMC matrix reinforcement. This result was also in a good accordance with the tensile results. 


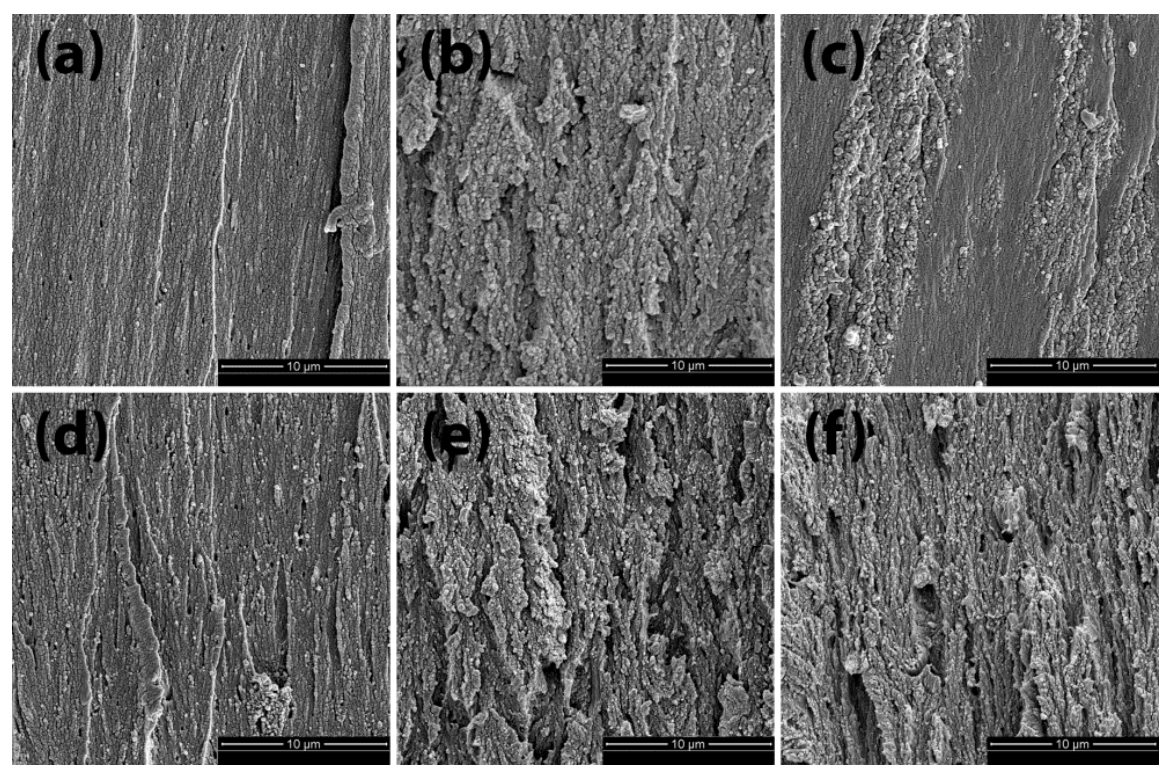

Figure 7. FE-SEM micrographs of fracture surface of (a) neat $\mathrm{CMC}$; (b) $\mathrm{CNC} 5 / \mathrm{CMC}$; (c) dCNC5/CMC; (d) cCNC1/CMC; (e) cCNC 5/CMC; and (f) cCNC10/CMC films.

The improved mechanical properties of CNC-reinforced polymer nanocomposites were primarily ascribed to the development in dispersion state of CNCs and interfacial bonding between CNCs and polymer matrix [14-22,25,26,28]. To explore the reinforcing mechanism, the fracture surface morphology of films was observed using FE-SEM (Figure 7). Neat CMC film exhibited featureless and smooth fracture surface (Figure 7a), which was well associated with its poor mechanical properties. It was reported that due to the hydrophilic characteristics of CNCs and the poor interfacial adhesion between $\mathrm{CNCs}$ and hydrophobic polymer, the CNCs aggregated and were present in the form of micro-sized flakes in hydrophobic polymer matrix [41-44]. However, in the compatible system through proper surface modification, the CNCs appeared as white dots, which evenly distributed in polymer matrix $[21,45,46]$. In the present study, both $\mathrm{CNCs}$ and mCNCs appeared as white dots without the formation of micro-sized flakes in the 
CMC matrix, suggesting the good compatibility between $\mathrm{CNCs} / \mathrm{mCNCs}$ and $\mathrm{CMC}$ matrix. Furthermore, more rough fracture surface was produced with the addition of $5 \mathrm{wt} \% \mathrm{CNCs}$ and mCNCs (Figures 7b, 7c and 7e). Among these films, the cCNC5/CMC film exhibited the most rough fracture surface, indicating the construction of the strongest interfacial interaction between cCNCs and CMC matrix. It was also observed that the dispersion state of mCNCs in CMC matrix was greatly dependent on their substitution degree. Very poor dispersion state was resulted from the dCNC5/CMC film (Figure 7c). Two distinctive phases, i.e., rough phase (corresponding to the filled fraction) and smooth phase (corresponding to the unfilled fraction) were clearly seen on the fracture surface. By contrast, the well dispersion state of cCNCs in CMC matrix was observed on the fracture surface of cCNC/CMC films with 1, 5 and 10 wt $\%$ cCNCs (Figures 7d-7e). The uniform dispersion state was well maintained even when $10 \mathrm{wt} \%$ cCNCs were incorporated.

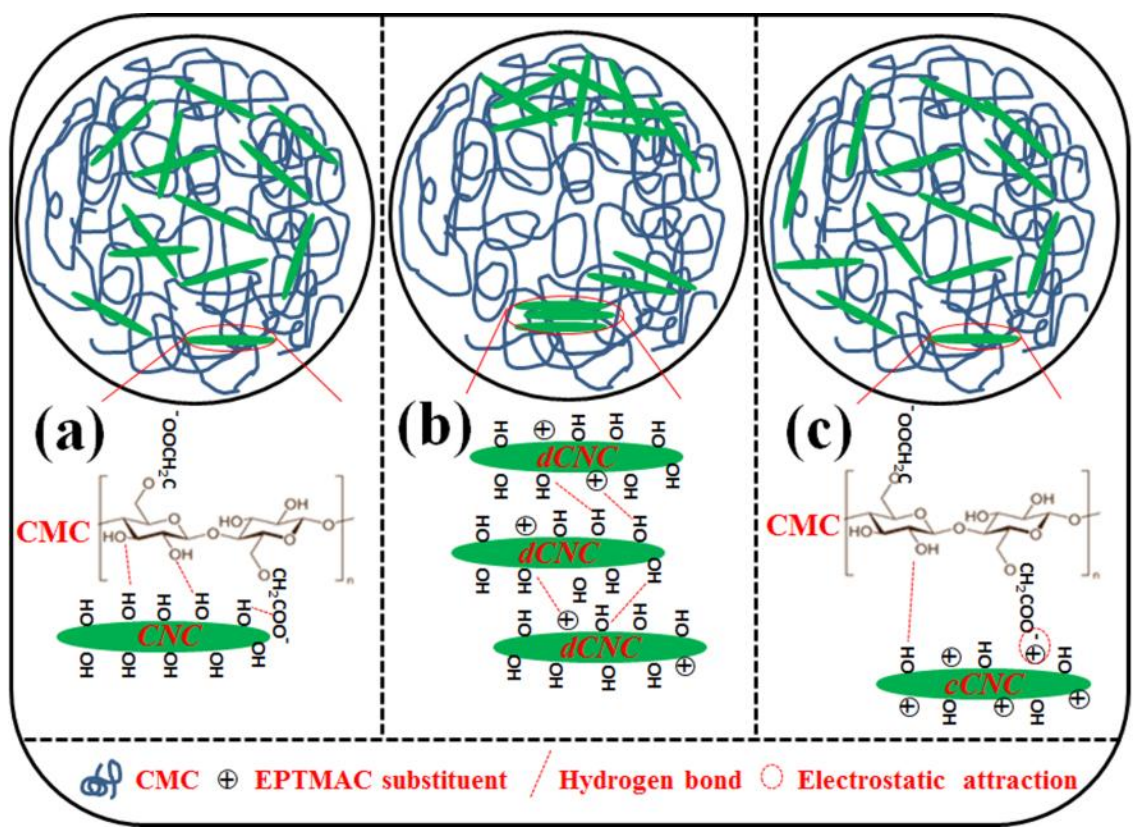

Figure 8. Schematic illustration on the reinforcing mechanisms for (a) $\mathrm{CNC} / \mathrm{CMC}$, (b) $\mathrm{dCNC} / \mathrm{CMC}$ and (c) cCNC/CMC films. 
Based on the above observations, distinctive reinforcing mechanisms for $\mathrm{CNC} / \mathrm{CMC}$, $\mathrm{dCNC} / \mathrm{CMC}$ and $\mathrm{cCNC} / \mathrm{CMC}$ films were reasonably proposed, as schematically illustrated in Figure 8. As known, CMC is an anionic cellulose derivate containing both hydroxyl and negatively charged carboxymethyl functional groups on the surface. The presence of such chemically reactive functional groups rendered $\mathrm{CMC}$ to easily form hydrogen bonding with the hydroxyl groups of CNCs (Figure 8a) [7-13]. Therefore, the reinforcement of CNCs in CMC matrix could be interpreted by taking account of the intrinsic characteristics of CNCs (e.g., nanoscale, large aspect ratio and high Young's modulus) and the formation of hydrogen bond at the CNC-CMC interface. After cationic modification, the presence of positively charged EPTMAC substituents on the surface of mCNCs was designed to 1) increase their dispersion state through electrostatic repulsion and 2) further enhance interfacial interaction through the construction of electrostatic attraction between the positively charged EPTMAC substituents of mCNCs and the negatively charged carboxylate group of CMC [47,48]. However, the experimental results showed that the substitution degree of mCNCs acted a critical role. At a low substitution degree, the dCNC aggregates were formed in aqueous solution due to the presence of predominant hydrogen bonding between the hydroxyl groups and the ammonium groups (Figures $3 \mathrm{c}$ and $3 \mathrm{~d}$ ). After mixing the $\mathrm{dCNC}$ suspension with $\mathrm{CMC}$, the created aggregates were imparted into its FFS as well as the final dried films, resulting in low-quality dCNC/CMC films with poor dispersion state (Figures $7 \mathrm{c}$ and $8 \mathrm{~b}$ ). Furthermore, due to the low substitution degree as well as the formation of aggregates, the amount of available ammonium groups in EPTMAC substituents to bind $\mathrm{CMC}$ molecular chains by electro static attraction was very limited, and thus the interfacial interaction between $\mathrm{dCNCs}$ and $\mathrm{CMC}$ matrix was relatively weak. When an external force was applied, the poor dispersion state easily induced stress concentration for rapid 
defect initiation at the interface. Meanwhile, the weak interfacial interaction further promoted the growth and propagation of crack from interface to the CMC matrix, and eventually caused the premature failure of the $\mathrm{dCNC} / \mathrm{CMC}$ films. At a high substation degree, the resultant cCNC suspension had improved dispersion state with low viscosity due to the occurrence of predominant electrostatic repulsion force as desired (Figures 3e and 3f). FE-SEM observations (Figures 7d-7f) confirmed that the uniform dispersion state of cCNCs in aqueous solution was successfully imparted into CMC films through solution casting method. More importantly, due to the introduction of more EPTMAC substituents on the surface of cCNCs, strong electrostatic attraction was built at the cCNC-CMC interface (Figure 8c). When an external force was applied, the synergetic effect of uniform dispersion state and strong interfacial bonding maximized the stress transfer from the $\mathrm{CMC}$ matrix to the rigid $\mathrm{cCNCs}$, resulting in superior reinforcement.

\section{Conclusions}

Surface cationization of CNCs was carried out through an alkali-activated etherification reaction with a cationic surfactant EPTMAC. Two types of mCNCs with different substitution degrees (i.e., cCNCs with substitution degree of 0.12 and dCNCs with substitution degree of 0.05 ) were obtained by controlling the water content of reaction system. The successful cationic modification was confirmed through different analytic techniques. It was found that the surface cationization of CNCs remarkably improved its thermal stability, and significantly affected its morphology, dispersion state and viscosity in aqueous solution depending on the substitution degree. At a low substitution degree, dCNCs were aggregated to bundles and clusters due to the formation of predominant hydrogen bonding between hydroxyl groups and quaternary ammonium groups of dCNCs. On the contrary, at a high substitution degree, cCNCs were well 
individualized and uniformly dispersed due to the formation of predominant electrostatic repulsion force between quaternary ammonium groups of cCNCs. Such different morphology, dispersion state and surface characteristics between cCNCs and dCNCs led to distinctive reinforcing phenomena in CMC films. Mechanical, dynamic mechanical and FE-SEM results indicated that cCNCs exhibited superior reinforcing capacity over CNCs and dCNCs at any loadings due to the improved dispersion state and interfacial bonding by electrostatic attraction between positively charged EPTMAC groups of cCNCs and negatively charged CMC molecular chains. However, dCNCs had worse reinforcing capacity than CNCs at high loadings due to the poor dispersion of $\mathrm{dCNCs}$ and the limited EPTMAC groups available for binding CMC molecular chains by electrostatic attraction. This study reported the preparation high performance nanocomposite films using cationized CNCs as reinforcing agent and anionic CMC biopolymer as matrix through the construction of strong electrostatic attraction at the interfaces, providing us a better understanding the critical role of surface chemistry of nanoscale fillers in the polymer reinforcement. The strategy reported here is also applicable for the preparation of high performance CNC-based hydrogels, drilling fluids, adhesive and etc.

\section{Acknowledgements}

This collaborative study was carried out with support from the National State Bureau of Forestry 948 Plan of China (Grant No. 2014-4-49), the Louisiana Board of Regents [LEQSF(2016-17) ENH-TR-01, LEQSF(2015-17)-RD-B-01], Korea National Institute of Forest Science, and the USDA National Institute of Food and Agriculture McIntire Stennis project [1000017]. 


\section{References}

(1) Griggs, D.; Stafford-Smith, M.; Gaffney, O.; Rockstrom, J.; Ohman, M. C.; Shyamsundar, P.; Steffen, W.; Glaser, G.; Kanie, N.; Noble, I. Policy: Sustainable development goals for people and planet. Nature 2013, 495, 305-307.

(2) Yu, L.; Dean, K.; Li, L. Polymer blends and composites from renewable resources. Prog. Polym. Sci. 2006, 31, 576-602.

(3) Wang, W.; Guo, Y.; Otaigbe, J. U. Synthesis, characterization and degradation of biodegradable thermoplastic elastomers from poly(ester urethane)s and renewable soy protein isolate biopolymer. Polymer 2010, 51, 5448-5455.

(4) Biswal, D. R.; Singh, R. P. Characterisation of carboxymethyl cellulose and polyacrylamide graft copolymer. Carbohydr. Polym. 2004, 57, 379-387.

(5) Zou, J.; Iyer, K. S.; Raston, C. L. Pd-sodium carboxymethyl cellulose nanocomposites display a morphology dependent response to hydrogen gas. Green Chem. 2012, 14, 906-908.

(6) Nadagouda, M. N.; Varma, R. S. Synthesis of Thermally Stable Carboxymethyl Cellulose/Metal Biodegradable Nanocomposites for Potential Biological Applications. Biomacromolecules 2007, 8, 2762-2767.

(7) Son, Y.-R.; Rhee, K. Y.; Park, S.-J. Influence of reduced graphene oxide on mechanical behaviors of sodium carboxymethyl cellulose. Compos. Part B Eng. 2015, 83, 36-42.

(8) Almasi, H.; Ghanbarzadeh, B.; Entezami, A. A. Physicochemical properties of starchCMC-nanoclay biodegradable films. Int. J. Biol. Macromol. 2010, 46, 1-5.

(9) Yadollahi, M.; Namazi, H.; Barkhordari, S. Preparation and properties of carboxymethyl cellulose/layered double hydroxide bionanocomposite films. Carbohydr. Polym. 2014, 108, 8390.

(10) Li, M.-C.; Wu, Q.; Song, K.; Cheng, H. N.; Suzuki, S.; Lei, T. Chitin Nanofibers as Reinforcing and Antimicrobial Agents in Carboxymethyl Cellulose Films: Influence of Partial Deacetylation. ACS Sustainable Chem. Eng. 2016, 4, 4385-4395. 
(11) Choi, Y.; Simonsen, J. Cellulose Nanocrystal-Filled Carboxymethyl Cellulose Nanocomposites. J. Nanosci. Nanotechnol. 2006, 6, 633-639.

(12) El Miri, N.; Abdelouahdi, K.; Barakat, A.; Zahouily, M.; Fihri, A.; Solhy, A.; El Achaby, M. BNFs reinforced with cellulose nanocrystals: Rheology of film-forming solutions, transparency, water vapor barrier and tensile properties of films. Carbohydr. Polym. 2015, 129, $156-167$.

(13) Oun, A. A.; Rhim, J.-W. Isolation of cellulose nanocrystals from grain straws and their use for the preparation of carboxymethyl cellulose-based nanocomposite films. Carbohydr. Polym. 2016, 150, 187-200.

(14) Favier, V.; Chanzy, H.; Cavaille, J. Y. Polymer Nanocomposites Reinforced by Cellulose Whiskers. Macromolecules 1995, 28, 6365-6367.

(15) Dufresne, A. Processing of Polymer Nanocomposites Reinforced with Polysaccharide Nanocrystals. Molecules 2010, 15, 4111-4128.

(16) Spinella, S.; Lo Re, G.; Liu, B.; Dorgan, J.; Habibi, Y.; Leclère, P.; Raquez, J.-M.; Dubois, P.; Gross, R. A. Polylactide/cellulose nanocrystal nanocomposites: Efficient routes for nanofiber modification and effects of nanofiber chemistry on PLA reinforcement. Polymer 2015, $65,9-17$.

(17) Girouard, N.; Schueneman, G. T.; Shofner, M. L.; Meredith, J. C. Exploiting colloidal interfaces to increase dispersion, performance, and pot-life in cellulose nanocrystal/waterborne epoxy composites. Polymer 2015, 68, 111-121.

(18) Kargarzadeh, H.; Sheltami, R. M.; Ahmad, I.; Abdullah, I.; Dufresne, A. Cellulose nanocrystal reinforced liquid natural rubber toughened unsaturated polyester: Effects of filler content and surface treatment on its morphological, thermal, mechanical, and viscoelastic properties. Polymer 2015, 71, 51-59.

(19) Dhar, P.; Bhasney, S. M.; Kumar, A.; Katiyar, V. Acid functionalized cellulose nanocrystals and its effect on mechanical, thermal, crystallization and surfaces properties of poly (lactic acid) bionanocomposites films: A comprehensive study. Polymer 2016, 101, 75-92. 
(20) Miao, C.; Hamad, W. Y. Alkenylation of cellulose nanocrystals (CNC) and their applications. Polymer 2016, 101, 338-346.

(21) Ansari, F.; Salajková, M.; Zhou, Q.; Berglund, L. A. Strong Surface Treatment Effects on Reinforcement Efficiency in Biocomposites Based on Cellulose Nanocrystals in Poly(vinyl acetate) Matrix. Biomacromolecules 2015, 16, 3916-3924.

(22) Gwon, J.-G.; Cho, H.-J.; Chun, S.-J.; Lee, S.; Wu, Q.; Li, M.-C.; Lee, S.-Y. Mechanical and thermal properties of toluene diisocyanate-modified cellulose nanocrystal nanocomposites using semi-crystalline poly(lactic acid) as a base matrix. $R S C A d v$. 2016, 6, 73879-73886.

(23) Lin, N.; Dufresne, A. Surface chemistry, morphological analysis and properties of cellulose nanocrystals with gradiented sulfation degrees. Nanoscale 2014, 6, 5384-5393.

(24) Hasani, M.; Cranston, E. D.; Westman, G.; Gray, D. G. Cationic surface functionalization of cellulose nanocrystals. Soft Matter 2008, 4, 2238-2244.

(25) Sirviö, J. A.; Honkaniemi, S.; Visanko, M.; Liimatainen, H. Composite Films of Poly(vinyl alcohol) and Bifunctional Cross-linking Cellulose Nanocrystals. ACS Appl. Mater. Interfaces 2015, 7, 19691-19699.

(26) Raquez, J.-M.; Murena, Y.; Goffin, A.-L.; Habibi, Y.; Ruelle, B.; DeBuyl, F.; Dubois, P. Surface-modification of cellulose nanowhiskers and their use as nanoreinforcers into polylactide: A sustainably-integrated approach. Compos. Sci. Technol. 2012, 72, 544-549.

(27) Tang, J.; Lee, M. F. X.; Zhang, W.; Zhao, B.; Berry, R. M.; Tam, K. C. Dual Responsive Pickering Emulsion Stabilized by Poly[2-(dimethylamino)ethyl methacrylate] Grafted Cellulose Nanocrystals. Biomacromolecules 2014, 15, 3052-3060.

(28) Tian, C.; Fu, S.; Habibi, Y.; Lucia, L. A. Polymerization Topochemistry of Cellulose Nanocrystals: A Function of Surface Dehydration Control. Langmuir 2014, 30, 14670-14679.

(29) Morandi, G.; Heath, L.; Thielemans, W. Cellulose Nanocrystals Grafted with Polystyrene Chains through Surface-Initiated Atom Transfer Radical Polymerization (SI-ATRP). Langmuir 2009, 25, 8280-8286. 
(30) Chen, J.; Lin, N.; Huang, J.; Dufresne, A. Highly alkynyl-functionalization of cellulose nanocrystals and advanced nanocomposites thereof via click chemistry. Polym. Chem. 2015, 6, $4385-4395$.

(31) Zaman, M.; Xiao, H.; Chibante, F.; Ni, Y. Synthesis and characterization of cationically modified nanocrystalline cellulose. Carbohydr. Polym. 2012, 89, 163-170.

(32) Cao, X.; Habibi, Y.; Lucia, L. A. One-pot polymerization, surface grafting, and processing of waterborne polyurethane-cellulose nanocrystal nanocomposites. J. Mater. Chem. 2009, 19, 7137-7145.

(33) Mansfield, S. D.; Meder, R. Cellulose hydrolysis -- the role of monocomponent cellulases in crystalline cellulose degradation. Cellulose 2003, 10, 159-169.

(34) Siqueira, G.; Bras, J.; Dufresne, A. New Process of Chemical Grafting of Cellulose Nanoparticles with a Long Chain Isocyanate. Langmuir 2010, 26, 402-411.

(35) Tingaut, P.; Hauert, R.; Zimmermann, T. Highly efficient and straightforward functionalization of cellulose films with thiol-ene click chemistry. J. Mater. Chem. 2011, 21, 16066-16076.

(36) Yang, H.; Yan, R.; Chen, H.; Lee, D. H.; Zheng, C. Characteristics of hemicellulose, cellulose and lignin pyrolysis. Fuel 2007, 86, 1781-1788.

(37) Wang, N.; Ding, E.; Cheng, R. Thermal degradation behaviors of spherical cellulose nanocrystals with sulfate groups. Polymer 2007, 48, 3486-3493.

(38) Cao, X.; Ding, B.; Yu, J.; Al-Deyab, S. S. Cellulose nanowhiskers extracted from TEMPO-oxidized jute fibers. Carbohydr. Polym. 2012, 90, 1075-1080.

(39) Ghannam, M. T.; Esmail, M. N. Rheological properties of carboxymethyl cellulose. $J$. Appl. Polym. Sci. 1997, 64, 289-301.

(40) Yasuda, K.; Armstrong, R. C.; Cohen, R. E. Shear flow properties of concentrated solutions of linear and star branched polystyrenes. Rheol. Acta 1981, 20, 163-178. 
(41) Arrieta, M. P.; Fortunati, E.; Dominici, F.; Rayón, E.; López, J.; Kenny, J. M. Multifunctional PLA-PHB/cellulose nanocrystal films: processing, structural and thermal properties. Carbohydr. Polym. 2014, 107, 16-24.

(42) Fortunati, E.; Armentano, I.; Zhou, Q.; Puglia, D.; Terenzi, A.; Berglund, L. A.; Kenny, J. M. Microstructure and nonisothermal cold crystallization of PLA composites based on silver nanoparticles and nanocrystalline cellulose. Polym. Degrad. Stab. 2012, 97, 2027-2036.

(43) Bagheriasl, D.; Carreau, P. J.; Dubois, C.; Riedl, B. Properties of polypropylene and polypropylene/poly(ethylene-co-vinyl alcohol) blend/CNC nanocomposites. Compos. Sci. Technol. 2015, 117, 357-363.

(44) Fortunati, E.; Armentano, I.; Zhou, Q.; Iannoni, A.; Saino, E.; Visai, L.; Berglund, L. A.; Kenny, J. M. Multifunctional bionanocomposite films of poly(lactic acid), cellulose nanocrystals and silver nanoparticles. Carbohydr. Polym. 2012, 87, 1596-1605.

(45) Azizi Samir, M. A. S.; Alloin, F.; Sanchez, J.-Y.; El Kissi, N.; Dufresne, A. Preparation of Cellulose Whiskers Reinforced Nanocomposites from an Organic Medium Suspension. Macromolecules 2004, 37, 1386-1393.

(46) Lin, N.; Huang, J.; Chang, P. R.; Feng, J.; Yu, J. Surface acetylation of cellulose nanocrystal and its reinforcing function in poly(lactic acid). Carbohydr. Polym. 2011, 83, 18341842.

(47) Wang, J.; Somasundaran, P. Adsorption and conformation of carboxymethyl cellulose at solid-liquid interfaces using spectroscopic, AFM and allied techniques. J. Colloid Interface Sci. 2005, 291, 75-83.

(48) Nie, L.; Liu, C.; Wang, J.; Shuai, Y.; Cui, X.; Liu, L. Effects of surface functionalized graphene oxide on the behavior of sodium alginate. Carbohydr. Polym. 2015, 117, 616 


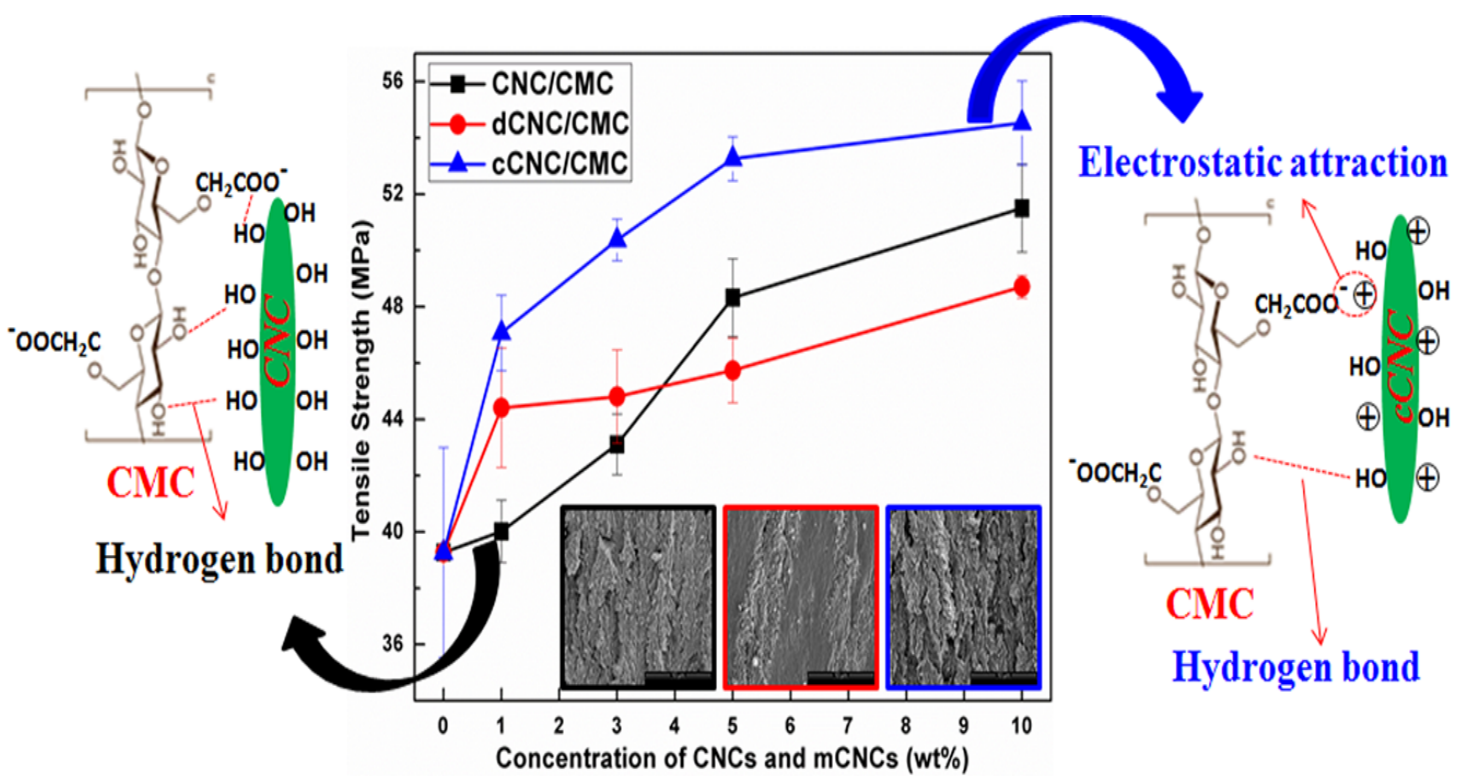

